LBNL-XXXx

ST/F TN-08/13

\title{
Design and Commissioning Plan for a Laser Heater for FERMI@elettra
}

\author{
A. Zholents, J. Qiang, M. Venturini, R. Wells, and R. Wilcox \\ Lawrence Berkeley National Laboratory, Berkeley CA, USA \\ S. Di Mitri , S. Spampinati, and G. Penco \\ Sincrotrone Trieste, Trieste (Italy)
}

\begin{abstract}
The purpose of a laser heater is to increase the electron beam uncorrelated energy spread as a way to control and ideally suppress the microbunching instability in the linac drive for x-rays FELs. We review the motivations for equipping FERMI with a laser heater and provide a specification for the basics parameters as well as a description of a practical layout including desired diagnostics provisions for both the electron and laser beams. We also outline some useful operational guidelines for commissioning.
\end{abstract}




\section{Disclaimer}

This document was prepared as an account of work sponsored by the United states Government. While this document is believed to contain correct information, neither the United states Government nor any agency thereof, nor the Regents of the University of California, nor any of their employees, makes any warranty, express or implied, or assumes any legal responsibility for the accuracy, completeness, or usefulness of any information, apparatus, product, or process disclosed, or represents that its use would not infringe privately owned rights. Reference herein to any specific commercial product, process, or service by its trade name, trademark, manufacturer, or otherwise, does not necessarily constitute or imply its endorsement, recommendation, or favoring by the United states Government or any agency thereof, or The Regents of the University of California. The views and opinions of authors expressed herein do not necessarily state or reflect those of the United States Government or any agency thereof, or The Regents of the University of California.

Ernest Orlando Lawrence Berkeley National Laboratory is an equal opportunity employer. 


\section{Introduction}

The concept of laser heater was proposed in [1] and soon after followed-up in [2] as an effective device for controlling the microbunching instability in the drive linac for x-rays FELs. The immediate action of a laser heater is to increase the uncorrelated energy spread of the electron beam after injection.

The effect of an increased energy spread on the microbunching instability is illustrated in Figure 1. The figure shows results from simulations of the final uncorrelated slice energy spread at the end of FERMI linac as a function of the initial uncorrelated energy spread at the beginning of the linac. Two sets of data are presented corresponding to two alternate FERMI lattices with one and two chicanes for bunch compression. The fairly large final energy spreads seen in the left part of the plots for both data sets at small values of the initial energy spread are due to the microbunching instability. In these simulations the microbunching instability is seeded by a random perturbation of the initial electron beam density meant to model the effect of shot noise. These initially small perturbations are amplified by the instability as the beam travels through the chicane(s) because of collective forces. The net result at the exit of the linac can be a beam with sizeable density modulation and unacceptably large energy spread. The error bars in the figure give the range of the energy spread values at the exit of the linac for various seeds used to generate the initial random perturbations.

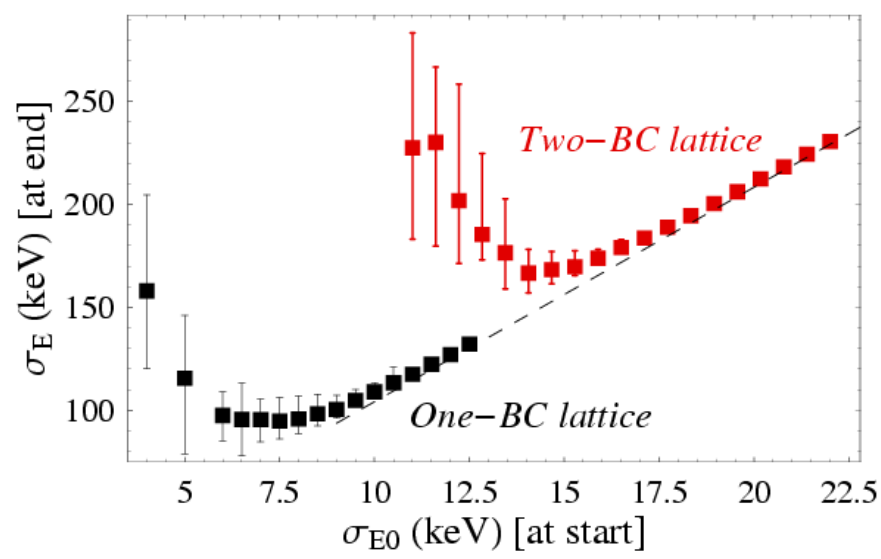

Figure 1. Uncorrelated rms energy spread for the beam at the exit of the linac as a function of that of the beam at injection for two alternate FERMI lattices with one and two bunch compressors $(B C)$. The error bars show the range of the energy spread resulting from various random realizations of the initial noise seeding the instability.

By increasing the energy spread in the beam at the start of the linac (x-axis of Figure 1) one promotes mixing of the electron trajectories within the bunch, which counters the clustering of the beam density caused by the instability. One can observe that by increasing the initial energy spread the microbunching instability gradually looses its 
strength as the mixing mechanism becomes more and more effective. However, continuing to increase the initial energy spread beyond a certain value becomes counterproductive as the final rms energy spread cannot be smaller than the product between the initial rms energy spread and the compression factor (dashed line in the figure). As a result, as we keep increasing the initial energy spread we observe the appearance of a fairly shallow minimum past which the final energy spread begins to grow. This is the point where the microbunching instability is effectively damped and where the laser heater should operate. The simulations showed in Fig.1 were carried out with a Vlasov solver [3,4] developed specifically to study the longitudinal beam dynamics in linacs.

The way the laser heater operates to increase the energy spread of an electron bunch is the following. The electron beam propagates along an undulator located inside a small chicane together with a longer laser pulse. An electron beam energy of $100 \mathrm{MeV}$, laser light wavelength of $800 \mathrm{~nm}$, an undulator period of $4 \mathrm{~cm}$ and the undulator parameter $K$ are chosen to yield a so-called FEL resonance condition when the laser light interacts with the electrons. During the interaction, some electrons gain energy and some electrons loose energy and, as a result, immediately after the undulator the electron bunch displays an energy modulation that reproduces the sinusoidal footprint of the laser pulse. However, because the laser intensity has a transverse Gaussian profile the amplitude of energy modulation varies depending on electron horizontal and vertical coordinates within each slice of the electron bunch. Past the undulator the electrons travel through the last two bending magnets of the chicane. The pathlengths of the electron trajectories through these magnets are different and depend on the electrons horizontal coordinate. Thus, at the end of the chicane the electrons in neighboring slices mix. This mixing destroys the regular sinusoidal pattern of energy modulation imposed by the laser pulse and creates a mix of electron energies within each slice of the electron bunch. The result is the appearance of a net increase of uncorrelated energy spread. Evidently, one important parameter defining the performance of the laser heater is the relative overlap of the laser light and the electron beam. For example, if the laser beam size at the focus $w_{0}$ is much larger than the electron beam size $\sigma_{x}$, then all the electrons within the same slice will get the same energy change independent from their radial off-set. The analysis in $[2,5,6]$ shows that the best result producing the most effective mix of the electron energies can be achieved when $w_{0} \approx 2 \sigma_{x}$. 


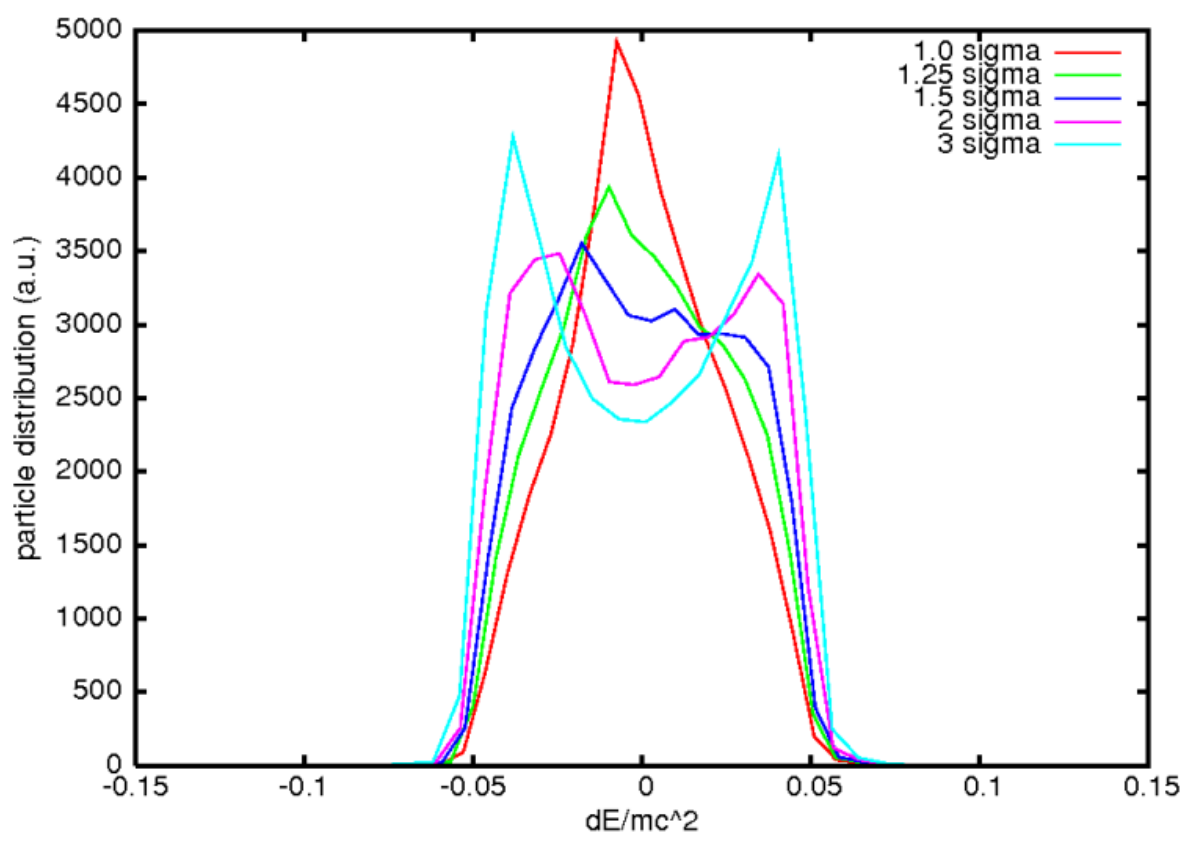

Figure 2. A set of histograms for the beam energy spread after FERMI laser heater obtained with IMPACT [8]. Different colors correspond to different ratio of $w_{0} / \sigma_{x}$ indicated in the top right corner of the plot. Laser peak power of $\sim 20 \mathrm{MW}$ was assumed in all cases.

In practice, the electron beam may not have identical transverse sizes in all slices along the electron bunch. In fact, according to [7] the FERMI injector has been designed to produce a particle distribution with a ramp in the peak current and a side effect of that is appearance of a rather significant variation of the bunch transverse sizes along the longitudinal coordinate. Therefore, the optimal choice of $w_{0}$ must be made such as to balance the variations in the energy spread produced by the laser heater along the electron bunch considering all slices within the electron bunch. To confirm this and to gain an understanding of what should be the optimal $w_{0}$ we performed simulations with various ratios of $w_{0} / \sigma_{x}$ and obtained a set of histograms for the energy spread after the laser heater chicane shown in Figure 2.

As discussed before, there will be a need to adjust the magnitude of the energy spread produced by the laser heater. One can do it by adjusting the laser beam peak power going into the undulator (without affecting the laser beam spot size). We estimate that the laser beam with peak power of the order of $2 \mathrm{MW}$ will produce the energy spread of the order of $10 \mathrm{keV}$. The requirement to the laser power scales quadratic with the projected energy spread. Thus, one may consider that $8 \mathrm{MW}$ laser could be sufficient to broaden the dynamic range in energy spread to $20 \mathrm{keV}$. Although $20 \mathrm{keV} \mathrm{rms}$ energy spread after the laser heater may seem to be excessive for a control on microbunching instability in the case of a one-BC lattice, we argue that it is needed for a safety margin. We also note that linac operation with two bunch compressors requires larger beam energy spread to get rid of the instability [8] (see Figure 1). We also consider that likely more laser beam power 
than predicted will be required considering known discrepancies between predicted energy modulation and measured energy modulation in "slicing" experiments at ALS and BESSY [9].

Finally we show in Figure 3 slice electron beam energy spread at the end of the spreader calculated using IMPACT code [8] using 1 billion macroparticles. In both cases $w_{0}$ was chosen approximately equal to the value of $\sigma_{x}$ averaged over all beam slices. The first and the second case in Figure 3 are different by assumed laser peak power. Four times smaller laser peak power was assumed in the second case compared to the first case. We explain the variation of the slice energy spread along the electron bunch seen in both cases by variation in the vertical and horizontal electron beam sizes along the electron bunch in the laser heater.
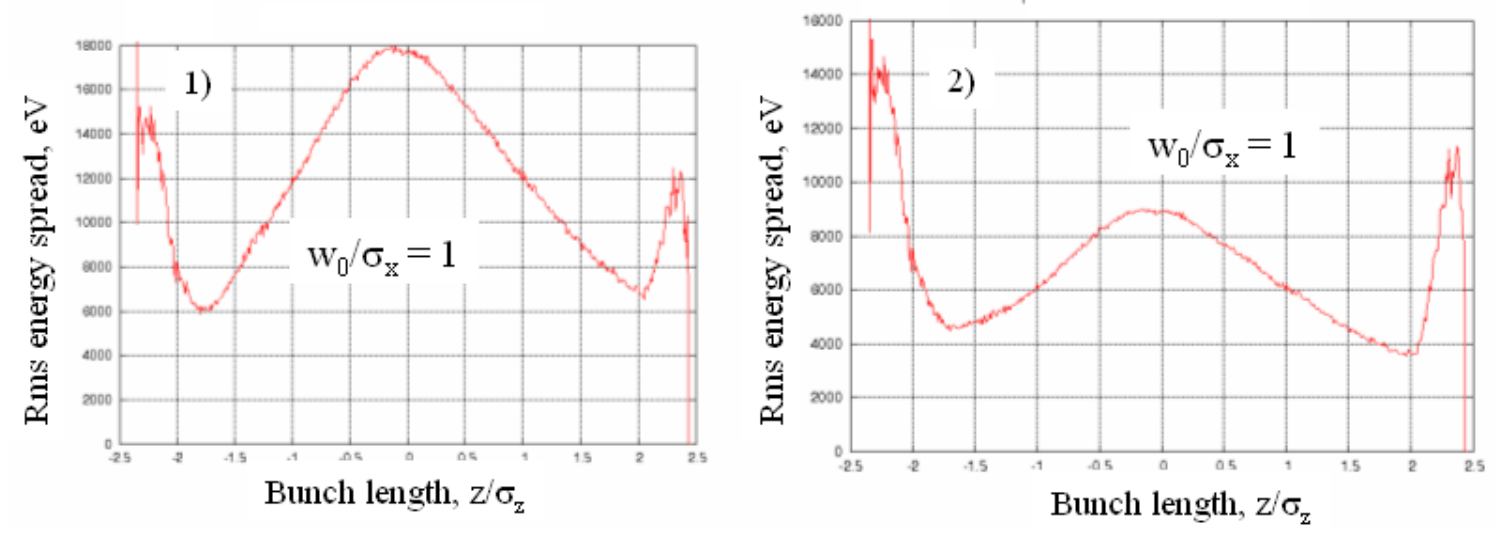

Figure 3. Slice energy spread at the end of the spreader. See text for a more detailed explanation of the two pictures 1) and 2).

\section{Design considerations and layout}

We propose to build laser heater as a system consisting of three regions (see Figure 4):

1) pre chicane beam transport

2) laser heater chicane

3) post chicane beam transport with a diagnostics branch line and a beam dump.

The first region, pre chicane beam transport, will include four quadrupoles for optics matching of the injector optics into the laser heater optics. These quadrupoles will provide the required flexibility to account for possible lattice mismatch in the upstream injector. Therefore we will build them with a maximum gradient of the magnetic field exceeding currently assumed value in the nominal lattice by at least a factor of two. We will include here additional elements for trajectory correction and diagnostic, namely, two steering magnets and three BPMs and will separate the last two BPMs by approximately 2-m long drift space that will allow us to continuously monitor the 
alignment of the electron beam with respect to the magnetic centers of the quadrupoles and made correction for a position and angle of the electron beam trajectory.

In the nominal lattice, the pre chicane quadrupoles provide minimum beta-functions (beam focus) in both transverse planes in the undulator center. One can take advantage of this setting and use three OTR monitors or fluorescent screens (to be defined latter which monitor is better) symmetrically spaced with respect to the beam crossover for emittance measurement in both transverse planes when laser heater chicane is switched off. As an alternative and complimentary diagnostics we consider using three wire scanners located next to the screens. These instruments could be more reliable than screens when the electron beam microbunching is taken place.

Pre chicane region is also convenient place for a location of the bunch phase monitor $(\mathrm{BPhM})$ which can be used for monitoring the arrival time of the electron bunch.

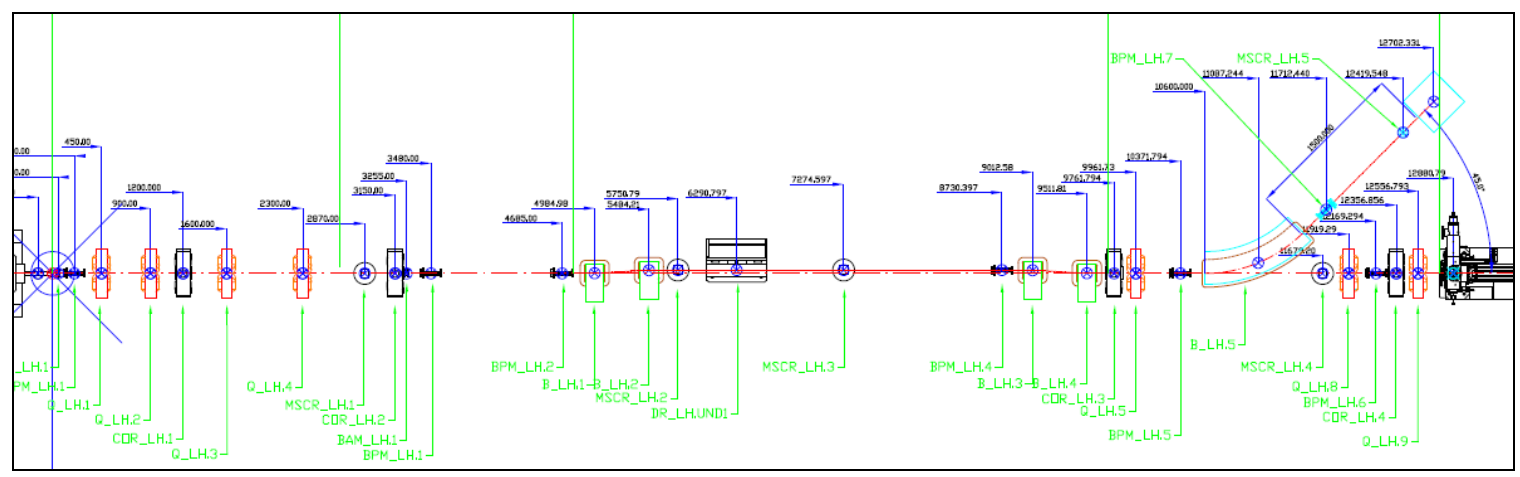

Figure 4. The laser heater system conceptual layout.

The second region, the laser heater chicane shown in Figure 5, will include four bending magnets forming a chicane where the two inner magnets will be positioned slightly off axis. The laser heater undulator will be positioned on a straight line trajectory between second and third bending magnet leaving a space to its right (see Figure 5) for additional instrumentation not specified here. The vacuum chamber in the laser heater chicane will be build to allow a straight path for the electron beam when chicane's magnets will be switched off. This is considered to be important for commissioning and for emittance measurements as discussed above. The laser beam will be brought into the undulator through a vacuum window located upstream of the second bending magnet. Similar window assembly will be used downstream of the third bend for diagnostics purposes (see discussion in Sec. 3.). The sizes for the upstream and downstream window will be chosen to accommodate foreseeable variations of the laser beam size $w_{0}$ at the focus. Electron beam and laser beam diagnostic inside the vacuum chamber of the laser heater will include two screens to be used for alignment of the laser and electron beams and one BPM to be also used for alignment and also for continuous monitoring of the electron beam trajectory. The downstream port after the third bending magnet will be used for diagnostic of the electron beam spontaneous emission from the undulator and for diagnostic of the laser beam (see discussion below and also in Sec. 3). A further development of this diagnostic channel should be considered at a later time. 


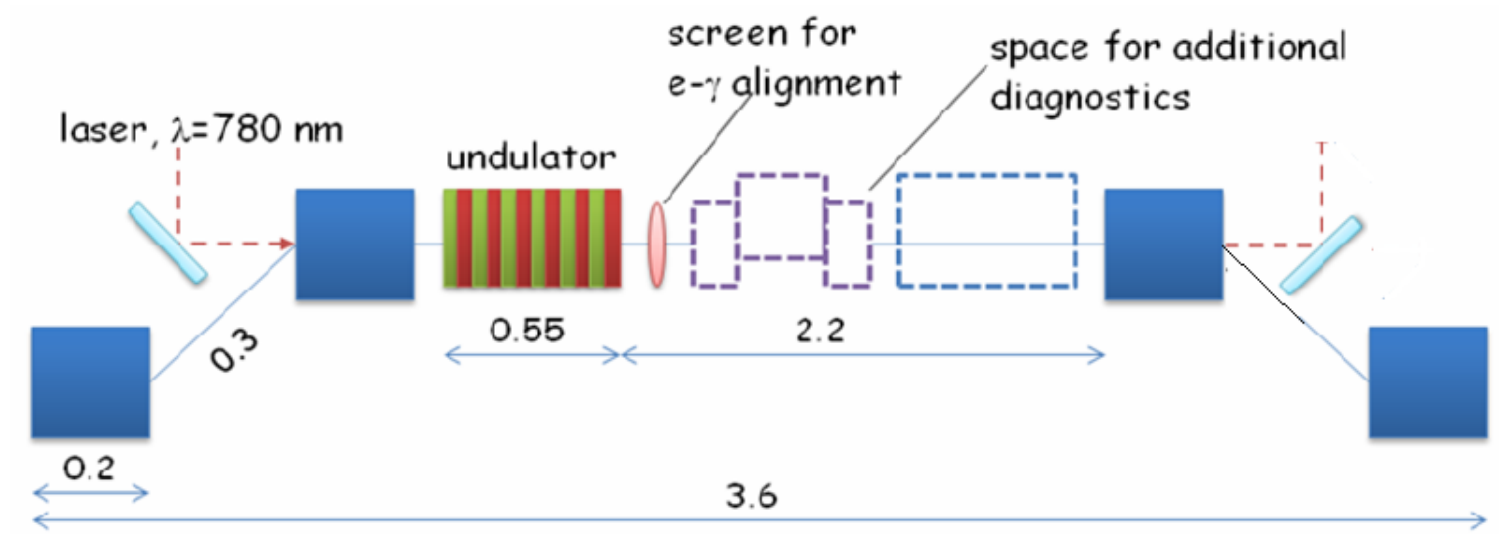

Figure 5. A schematic of the laser heater chicane. All dimensions are given in meters and for orientation purpose.

We make the following assumptions for the laser: the laser system should be capable of delivering power up to $40 \mathrm{MW}$ (see discussion above), $\mathrm{a} \sim 20 \mathrm{ps}$ pulse width and $\mathrm{M}^{2}$ factor less than 1.2. A capability to control laser pulse width, chirp, and phase front, and a capability to maintain stable alignment over the laser beam transport to the front of the laser heater "laser beam control system" shown in Figure 6 is also assumed.

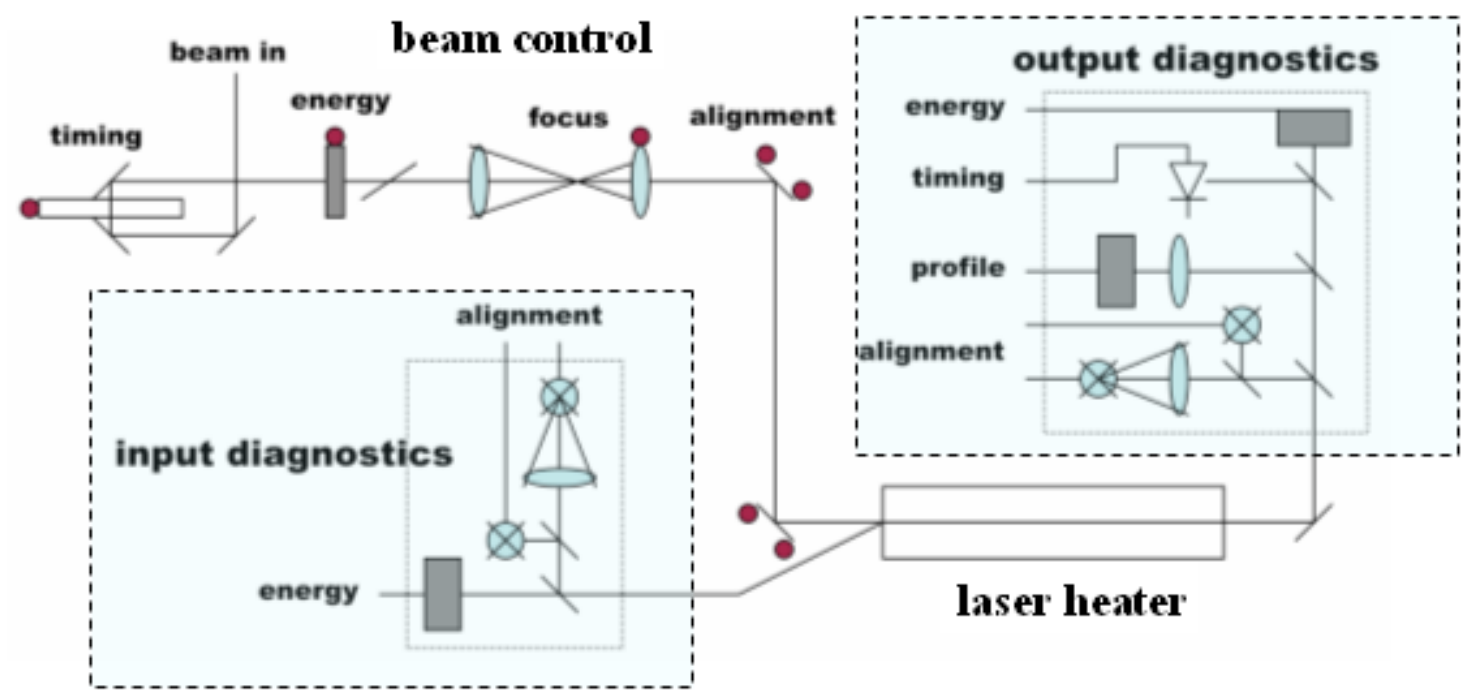

Figure 6. Laser beam control system block diagram. Optical components in the laser beam control subsystem include energy, timing, focusing and alignments controls.

The third region, the post chicane beam transport with a diagnostics branch line and a beam dump, contains a straight line for a beam transport into the following linac and a side line for a beam dump and energy spectrometer. The straight beam transport line will 
include three quadrupoles for beam focusing, two steering magnets for the trajectory correction, and two BPMs for monitoring of the electron beam orbit. It will also have one OTR or fluorescent screen to be used for the emittance measurement and trajectory alignment. The side beamline will include bending magnet and a quadrupole after the bending magnet (not shown in Figure 4). This beamline will serve as the intermediate beam dump during injector commissioning and as an energy spectrometer. In its spectrometer option, two quadrupoles, one upstream to the magnet and one downstream to the magnet will be used to maximizes the dispersion and minimize the horizontal betafunction at the OTR monitor upstream of the dump. To enhance convenience of the operation of this side beamline, BPM will also be used upstream of the OTR monitor.

The specifications for magnetic elements, i.e. quadrupoles, bending magnets, steering magnets, and required tolerances are reported in Tables 1-3.

Table 1. Dipole magnet requirements and tolerances.

\begin{tabular}{|c|c|c|c|c|c|}
\hline \# of magnets & \multicolumn{4}{|c|}{4} & \\
\hline Curvature Angle & \multicolumn{4}{|c|}{0.060900} & $\mathrm{rad}$ \\
\hline Curvature Radius & \multicolumn{4}{|c|}{3.284} & $\mathrm{~m}$ \\
\hline Magnetic Arclength & \multicolumn{4}{|c|}{200.0} & $\mathrm{~mm}$ \\
\hline $\begin{array}{l}\text { Magnetic Field at magnet } \\
\text { centre at } 0.095 \mathrm{GeV}\end{array}$ & \multicolumn{4}{|c|}{0.0965} & $\mathrm{~T}$ \\
\hline $\begin{array}{l}\text { Integrated magnetic field at } \\
0.095 \mathrm{GeV}\end{array}$ & \multicolumn{4}{|c|}{0.0193} & $\mathrm{Tm}$ \\
\hline $\begin{array}{l}\text { Max. integrated magnetic field } \\
\text { at } 0.120 \mathrm{GeV}\end{array}$ & \multicolumn{4}{|c|}{0.0244} & $\mathrm{Tm}$ \\
\hline $\begin{array}{l}\text { Residual integrated magnetic } \\
\text { field at } \mathrm{I}=0 \mathrm{~A}\end{array}$ & \multicolumn{4}{|c|}{$\leq 1$} & $\mathrm{Gm}$ \\
\hline Magnets Type & \multicolumn{4}{|c|}{ Rectangular } & \\
\hline Total Gap & \multicolumn{4}{|c|}{42} & $\mathrm{~mm}$ \\
\hline Magnet Name & B_LS.1 & B_LS.2 & B_LS.3 & B_LS.4 & \\
\hline $\begin{array}{l}\text { Field Homogeneity: } \\
\left|\Delta \mathrm{b}_{0} / \mathrm{b}_{0}\right| \text { in the range } \mathrm{x}= \pm 25 \\
\mathrm{~mm} \text { and } \mathrm{B}=0.012-0.122 \mathrm{~T}\end{array}$ & 1.60 & 1.50 & 1.80 & 2.10 & $\%$ \\
\hline $\begin{array}{l}\text { Field Integral Homogeneity: } \\
\mid \Delta \mathrm{U} / \mathrm{i} \text { in the range } x= \pm 25 \mathrm{~mm} \\
\text { and } \mathrm{B}=0.012-0.122 \mathrm{~T}\end{array}$ & 1.85 & 1.75 & 2.05 & 2.35 & $\%$ \\
\hline $\begin{array}{l}\text { Quadrupolar field component: } \\
\left|\mathrm{b}_{1} / \mathrm{b}_{0}\right| \text { at } \mathrm{R}= \pm 20 \mathrm{~mm}\end{array}$ & 6.1 & 1.3 & 1.1 & 5.0 & $\%$ \\
\hline $\begin{array}{l}\text { Pk-to-pk field integral } \\
\text { variation for all the } 4 \text { magnets } \\
\text { in the range } B=0.012-0.122 \mathrm{~T} \text {, } \\
\text { at same current }\end{array}$ & \multicolumn{4}{|c|}{1} & $\%$ \\
\hline
\end{tabular}


Table 2. Quadrupole magnet requirements and tolerances.

\begin{tabular}{|l|c|c|}
\hline \# of magnets & 8 & \\
\hline Integrated Gradient Range & $0.02-0.6$ & $\mathrm{~T}$ \\
\hline $\begin{array}{l}\text { Sum of all integrated normal } \\
\text { and skew multipoles beyond } \\
\mathrm{n}=1 \text { (main component) up to } \\
\mathrm{n}=9 \text { at } \mathrm{R}= \pm 20 \mathrm{~mm}\end{array}$ & 1.2 & $\%$ \\
\hline $\begin{array}{l}\text { Sextupolar field component: } \\
\left|\mathrm{b}_{2} / \mathrm{b}_{1}\right| \text { at } \mathrm{R}= \pm 20 \mathrm{~mm} \text { in the } \\
\text { specified range }\end{array}$ & 0.6 & $\%$ \\
\hline $\begin{array}{l}\text { Maximum residual } \\
\text { integrated gradient at I=0 A }\end{array}$ & 11 & $\mathrm{G}$ \\
\hline $\begin{array}{l}\text { Maximum residual } \\
\text { integrated field at I=0 A }\end{array}$ & 0.2 & $\mathrm{Gm}$ \\
\hline
\end{tabular}

Table 3. Steering magnet requirements and tolerances.

\begin{tabular}{|l|c|c|}
\hline \# of magnets & 8 & \\
\hline Maximum angular kick & 3.0 & $\mathrm{mrad}$ \\
\hline $\begin{array}{l}\text { Maximum integrated } \\
\text { magnetic field integrated }\end{array}$ & 10.5 & $\mathrm{Gm}$ \\
\hline $\begin{array}{l}\text { Maximum } \\
\text { quadrupole normal and skew } \\
\text { component at } \mathrm{R}= \pm \mathbf{1 5} \mathrm{mm}, \mathrm{I}_{\mathbf{1}} / \mathrm{I}_{0}\end{array}$ & 1.5 & $\%$ \\
\hline $\begin{array}{l}\text { Sum of all integrated normal } \\
\text { and skew multipoles beyond } \\
\mathbf{n}=0 \text { (main component) up to } \\
\mathbf{n = 9} \text { at } \mathrm{R}= \pm \mathbf{1 5} \text { mm }\end{array}$ & 1.5 & $\%$ \\
\hline Magnet Type & C-type & \\
\hline Total gap & 42 & $\mathrm{~mm}$ \\
\hline
\end{tabular}

\section{Guidelines for the development and installation of the Laser Heater}

\subsection{Commissioning objectives and strategy}

The main objective of the laser heater commissioning is to obtain a full functionality of this instrument as described in the previous section including functionality of additional diagnostics associated with it. This will require establishing electron beam and laser beam trajectories through undulator with spatial and time overlap of both beams, establishing control over electron and laser beam sizes in the undulator and adjusting them to obtain the nominal values, and, finally, characterization of the laser heater process. These goals can be further subdivided into several steps listed here in a logical order (and further elaborated in 3.2):

1) Assemble and test all equipment prior to installation in the accelerator tunnel. 
2) Assemble all equipment in the accelerator tunnel and test it without electron beam.

3) Establish unrestricted electron beam pass into the intermediate dump with all magnetic elements being switched off except the final bending magnet leading to the dump.

4) Characterize pulse-to-pulse jitter of the electron beam trajectory in two transverse planes after injector to be sure that it is consistent with required tolerance, i.e less than 30 micron rms trajectory jitter inside the undulator.

5) Turn on quadrupole lenses one at a time and perform beam based alignment for each quadrupole individually.

6) Turn on four quadrupoles upstream of the chicane and tweak them to obtain nominal beam size at a focus and nearby OTR monitors or fluorescent screens.

7) Measure electron beam profile using wire scanners and cross calibrate these measurements with measurements obtained using OTR monitors or fluorescent screens.

8) Refine beam focusing based on better understanding of the beam sizes.

9) Characterize pulse-to-pulse jitter of the electron beam trajectory in two transverse planes at the focus and make sure that it is consistent with required tolerance and prior measurements without quadrupoles turned on.

10) Turn on chicane magnets and establish unrestricted electron beam pass into the intermediate dump.

11) Characterize pulse-to-pulse jitter of the electron beam trajectory after chicane with chicane magnets turned on.

12) Turn on quadrupoles before and after bending magnet leading to the dump following the same procedure described in items 5 through 8 .

13) Calibrate electron beam energy using bending magnet leading to the dump.

14) Do beam based calibration of all BPMs and establish "golden" orbit.

15) Without the electron beam establish unrestricted laser beam pass through chicane into diagnostics hutch behind exit window.

16) Measure pulse-to-pulse jitter of the laser beam trajectory with laser beam trajectory feedback system turned off.

17) Measure pulse-to-pulse jitter of the laser beam trajectory with laser beam trajectory feedback system turned on and make sure that it is consistent with required tolerance.

18) Establish laser beam focus in the center of the undulator vacuum chamber.

19) Measure laser beam spot size at several location inside and outside of the vacuum chamber and adjust it to be in agreement with calculated sizes based on consideration of the electron beam size at the undulator center.

20) Measure and adjust polarization of the laser light.

21) Establish "golden" path for the laser beam.

22) Install undulator and close undulator jaws to a designed gap.

23) Re-establish "golden" orbit for the electron beam. 
24) Observe spontaneous emission of electrons in undulator and adjust the undulator gap to obtain a design spectrum.

25) Align laser beam to achieve spatial overlap with the electron beam in both transverse plains at the focus and establish a new "golden" path for the laser beam.

26) Measure timing jitter for the arrival time of the electron bunch using $\mathrm{BPhM}$ and make sure that it is consistent with required tolerance.

27) Measure timing jitter for the laser pulse arrival time using fast photodiode and make sure that it is consistent with required tolerance.

28) Achieve overlap in time for the pulse of spontaneous emission and for the laser pulse by adjusting time delay of the laser pulse.

The next logical step is characterization of the performance of the laser heater that requires measuring of the uncorrelated energy spread as function of the laser beam peak power and laser beam spot size in the undulator. A vertical deflecting cavity has its natural location downstream BC1. Since BC1 magnetic chicane will be with a movable chamber allowing the uncompressed beam to go straight, we should be able to measure the beam heating process also for the uncompressed bunch. By deflecting the electron bunch in vertical direction, one should be able to measure slice energy spread using the downstream energy spectrometer. We estimate that energy resolution of $5 \mathrm{keV} \mathrm{rms}$ can be obtained there in the case of the slice normalized electron beam emittance of 2 micron. The electron beam would need to be delivered into post $\mathrm{BC} 1$ area and it would require commissioning of the post chicane beam transport and downstream linac sections connecting the laser heater chicane and $\mathrm{BC} 1$.

As a back-up option, we suggest using the available free space in the laser heater chicane and temporarily move there the deflecting cavity. In this case the electron beam dump downstream of the laser heater chicane could be used as a spectrometer and for measurements of the beam slice energy spread. We estimate that energy resolution better than $4 \mathrm{keV}$ rms can be obtained there in the case of the slice normalized electron beam emittance of 2 micron.

\subsection{Commissioning process}

Here we will discuss in more detail several items listed above. We will skip detail discussion for items that seem to be self explanatory.

Step 1): Control assembly of all equipment, i.e.:

a) assemble all supporting structures, quadrupole lenses, bending magnets, steering magnets, vacuum chambers, vacuum pumps and monitors, vacuum windows, diagnostic elements including OTR monitors, fluorescence screens, BPMs, BPhM, wire scanners, and a beam dump;

b) obtain design vacuum;

c) connect all magnetic elements to respective power supplies and controls and check polarity of magnetic field and functionality of all controls;

d) equip in-vacuum diagnostics with external equipment, i.e. optics, cameras, controls and check their functionality; 
e) assemble three laser beam subsystems, i.e. laser beam control, laser beam input diagnostics, and laser beam output diagnostics, and check their functionality.

Step 2): Install all equipment in the linac tunnel and repeat everything what was described in Step 1); integrate equipment with undulator and its supporting structure. Then, remove undulator until Step 22) of commissioning restricting its exposure to excessive radiation.

Step 3): Use steering magnets upstream to the laser heater and bending magnet leading to the dump to steer the electron beam into the dump; record current position of the electron beam on all monitors.

Step 4): Using one quadrupole at a time observe steering of the electron beam trajectory with respect to the recorded positions produced by a quadrupole when it is powered at a maximum strength. Attempt to align each quadrupole to minimize its steering.

Step 5): After setting quadrupole strength at design values measure electron beam size at the downstream OTR monitors or fluorescent screens. Following tuning procedure (to be defined) achieve desirable beam focusing that will result in electron beam matching from the injector into the laser heater.

Step 6): Measure electron beam size with wire scanners and compare these measurements with electron beam size measurements obtained with OTR monitors or fluorescent screens. Then decide regarding relative measurement accuracy and attempt to extract information about electron beam projected emittance in both transverse planes. We anticipate that the transverse space charge forces will have only a mild impact on the beam sizes as one can see in Figure 8.

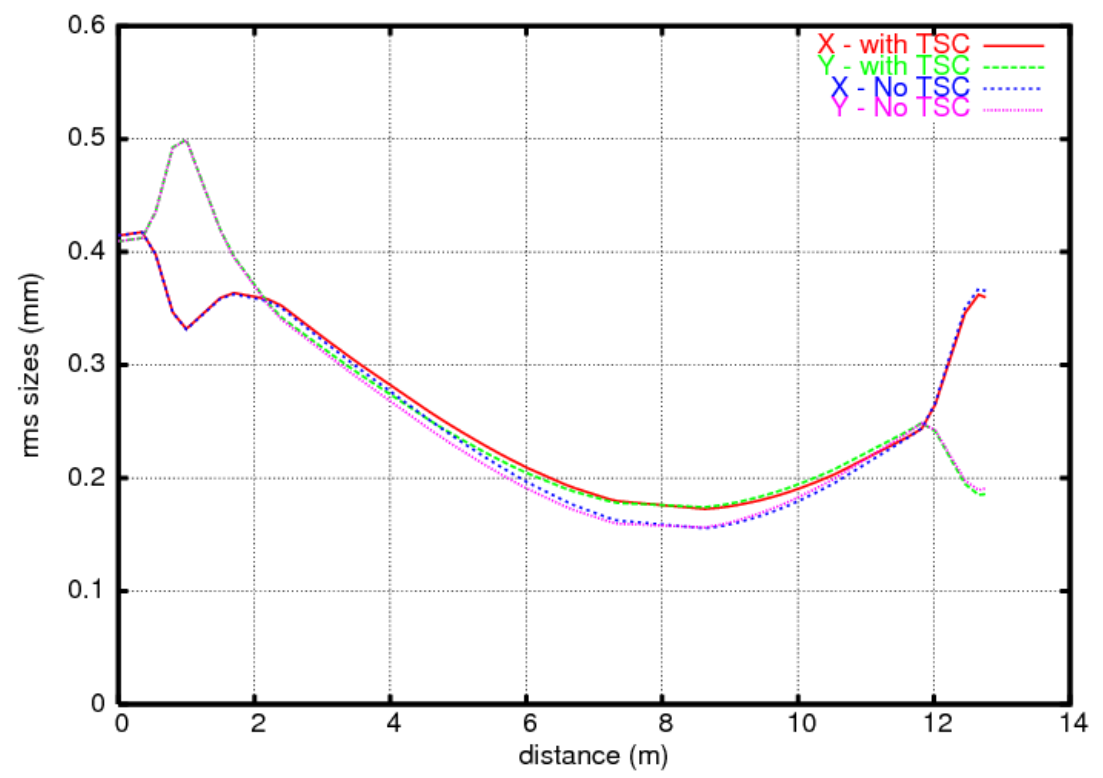


Figure 8. Electron beam size in the laser heater with laser heater chicane turned off with and without effect of the transverse space charge (TSC). Different lines are explained in the legend in the top right corner.

Step 7): Repeat measurement of the pulse-to-pulse jitter of the electron beam trajectory to make sure that matching quadrupoles do not introduce additional jitter.

Step 8): Calibrate electron beam energy (resolution limited) using measurements of the electron beam position in the middle of the chicane and at the entrance of the dump.

Step 9): Calibrate BPMs by comparing their measurements to electron beam trajectory measured with OTR monitors or fluorescent screens and establish reference (golden) orbit.

Step 10): Achieve co-linearity of the electron beam and laser beam trajectories through undulator and along the straight line between the second and third bending magnets of the chicane observing both beams on fluorescent screens inside the vacuum chamber and in the output diagnostics using spontaneous emission of the electrons in the undulator. Since the two signals are very different in energy, check the alignment of the laser beam with respect to the spontaneous emission by alternating filters using motorized filter holders to adjust both signals within the same sensitivity range of the diagnostics. Record a reference (golden) laser beam path using the output laser beam diagnostic and the input laser beam diagnostics that receives a reflection from the input vacuum window. In principle, using only the output laser beam diagnostics should be sufficient, but we will install also the input laser beam diagnostics in case if possible radiation darkening of the vacuum windows will makes it necessary to monitor this effect and compensate. The same effect may also bias beam alignment sensed through the output diagnostics. Additionally, the input laser beam diagnostics will enable a quick realignment of the laser beam when it is so misaligned as to not transmit through the laser heater at all.

Step 11): Use a fast oscilloscope to coarsely time spontaneous emission and laser pulse, alternately observing them on a high bandwidth photodiode. Adjust a time delay line to achieve timing overlap of two signals.

Acknowledgement. Work supported by Department of Energy Contract No. DE-AC0205CH11231.

\section{References}

1. E.L. Saldin, E.A. Schneidmiller, and M.V. Yurkov, DESY Report No. TESLA-FEL 2003-03, 2003.

2. Z. Huang, et al., Phys. Rev. ST -Accel. and Beams, V.7, 074401, (2004).

3. M. Venturini, Phys. Rev. ST - Accel. and Beams 10, 104401 (2007).

4. M. Venturini and A. Zholents, Nucl. Instr. and Meth. A (2008) doi:10.1016/j.nima.2008.04.036 
5. A. Zholents, FERMI@Elettra laser heater: requirements for the laser peak power, CBP Tech. Note - 371, (2007).

6. S. Spampinati, Modeling of the Laser Heater in FERMI@elettra, Fermi Tech Note, February 2007.

7. FERMI@Elettra Conceptual Design Report (CDR), January 2007.

8. J Ji Qiang, Ilya V. Pogorelov, Robert D. Ryne, Parallel Beam Dynamics Simulation Tools for Future Light Source Linac Modeling, in proceedings of PAC07 conference, June 25-29, Albuquerque, p. 3522, 2007.

9. S. Khan et al., Femtosecond undulator radiation from sliced electron bunches, Phys. Rev. Lett., 97, 074801(2006). 